\title{
POLAR spacecraft observations of helium ion angular anisotropy in the Earth's radiation belts
}

\author{
W. N. Spjeldvik ${ }^{1}$, T. A. Fritz ${ }^{2}$, J. Chen ${ }^{2}$, R. B. Sheldon ${ }^{2}$ \\ ${ }^{1}$ Physics Department, Weber State University, 2508 University Circle, Ogden, Utah 84408-2508, USA \\ E-mail:WSpjeldvik@CC.Weber.edu \\ ${ }^{2}$ Center for Space Physics, Boston University, 745 Commonwealth Avenue, Boston, MA 02215, USA
}

Received: 19 June 1998 / Revised: 30 October 1998 / Accepted: 16 November 1998

\begin{abstract}
New observations of energetic helium ion fluxes in the Earth's radiation belts have been obtained with the CAMMICE/HIT instrument on the ISTP/GGS POLAR spacecraft during the extended geomagnetically low activity period April through October 1996. POLAR executes a high inclination trajectory that crosses over both polar cap regions and passes over the geomagnetic equator in the heart of the radiation belts. The latter attribute makes possible direct observations of nearly the full equatorial helium ion pitch angle distributions in the heart of the Earth's radiation belt region. Additionally, the spacecraft often re-encounters the same geomagnetic flux tube at a substantially offequatorial location within a few tens of minutes prior to or after the equatorial crossing. This makes both the equatorial pitch angle distribution and an expanded view of the local off-equatorial pitch angle distribution observable. The orbit of POLAR also permitted observations to be made in conjugate magnetic local time sectors over the course of the same day, and this afforded direct comparison of observations on diametrically opposite locations in the Earth's radiation belt region at closely spaced times. Results from four helium ion data channels covering ion kinetic energies from 520 to $8200 \mathrm{KeV}$ show that the distributions display trapped particle characteristics with angular flux peaks for equatorially mirroring particles as one might reasonably expect. However, the helium ion pitch angle distributions generally flattened out for equatorial pitch angles below about $45^{\circ}$. Significant and systematic helium ion anisotropy difference at conjugate magnetic local time were also observed, and we report quiet time azimuthal variations of the anisotropy index.
\end{abstract}

Key words. Magnetospheric physics (energetic particles, trapped; magnetospheric configuration and dynamics; plasmasphere).

Correspondence to: W. N. Spjeldvik

\section{Introduction}

The Earth's radiation belts have been studied since the beginning of the space era (e.g., summaries by Roederer, 1970; Schulz and Lanzerotti, 1974; Spjeldvik and Rothwell, 1985; Walt, 1994), and ions heavier than protons have become recognized as important components of the terrestrial space environment (e.g., MogroCampero, 1972; Hovestadt et al., 1972; Krimigis and Van Allen, 1976; Spjeldvik, 1979; Spjeldvik and Fritz, 1983; Spjeldvik, 1996a; and references therein). Theoretical attention to expected angular distributions of geomagnetically confined ions was given as early as in the 1960s and 1970s (e.g., Northrop and Teller, 1960; Hamlin et al., 1961; Dungey, 1966; Roederer and Schulz, 1971; Stern, 1971; Schulz, 1972; Kivelson and Southwood, 1975a,b; Southwood and Kivelson, 1975; and references therein).

Except for times of sudden impulsive injections or shock-like events, radiation belt energetic ions at $\mathrm{MeV}$ kinetic energies convect so slowly across geomagnetic field lines that the long time scale diffusive transport dominates dynamic behavior. To evaluate steady state radial and pitch angle distributions of helium ions in the interior of the radiation belts, one must consider a balance between transport and losses (e.g. Nakada and Mead, 1965; Cornwall, 1972). In an early Russian work, Tverskoy (1971) modeled the $L$-shell location of the equatorially mirroring peak fluxes to occur where the characteristic time scales of cross- $L$ transport and of local losses coincide. For helium ions at $\mathrm{E} \sim 1 \mathrm{MeV}$, the effective local loss time scale is similar to, or smaller than, the cross- $\mathrm{L}$ diffusive transport time scale at $L<3.5$ (e.g. Spjeldvik and Fritz, 1978, 1981) at the geomagnetic equator, and the $L$-shell range where this is true expands towards higher $L$-shells with lower equatorial pitch angles. This is so because charged particles with low equatorial pitch angles encounter a denser bounceaveraged exosphere, and thus suffer comparatively 
greater collisional interactions and losses (e.g., quantitative evaluation by Smith and Bewtra, 1978; Smith et al., 1981).

Moreover, ions with different pitch angles observed at $L=3$ (for example) come from different parts of the ion spectrum in the source population at higher $L$-shells (since the parallel ion kinetic energy adiabatically varies as $L^{-2}$ while the perpendicular energy varies as $L^{-3}$ in the dipole B-field approximation), and this couples the observable radiation belt ion anisotropy with both the outer zone source spectrum and the energy and pitch angle dependent cross-field diffusive transport rate. It is therefore to be expected that the ion pitch angle distributions within the radiation belts should be determined by the combination of pitch angle dependent source mechanisms, by pitch angle dependent loss processes, by the cross- $L$ transport characteristics, and to some extent by electric and magnetic $L$-shell splitting in the interior of the radiation belts.

It is known that plasma waves in the whistler mode help control the structure of the electron radiation belts (i.e., Lyons and Thorne, 1973), and plasma waves in the ion-cyclotron mode are certainly important for protons and other ions in the lower $\mathrm{KeV}$ energy range (e.g., Taylor and Lyons, 1976; Joselyn and Lyons, 1976; and others), yet there is little reliable information to unambiguously establish an importance of wave scattering of $\mathrm{MeV}$ ions. Part of the reason for this has been the limited availability of detailed pitch angle distributions of different ion species in the central parts of the radiation belts where these ions are abundant. The observational findings presented herein may help remedy this empirical dearth.

Except for large injection events and magnetic shock transitions through the magnetosphere, helium ions can diffusively populate the radiation belts from an external source region such as the solar wind and solar energetic particles (e.g., Cornwall, 1972; Schulz and Lanzerotti, 1974; Lyons and Evans, 1976; Fritz and Spjeldvik, 1978, 1979, 1981; Spjeldvik and Fritz, 1978, 1981; Sheldon and Hamilton, 1993). Additionally, such ions (both ${ }^{3} \mathrm{He}$ and ${ }^{4} \mathrm{He}$ ) can also be generated in situ in the inner radiation zone by local nuclear interactions (e.g., Chen et al., 1994, 1996a,b; Pugacheva et al., 1996; Selesnick and Mewaldt, 1996; Spjeldvik et al., 1996; Gusev et al., 1996; and references therein). There have been several modeling studies of geomagnetically trapped energetic helium ions (e.g., Tverskoy, 1971; Cornwall, 1972; Krimigis and Van Allen, 1976; Fritz and Spjeldvik, 1978, 1979, 1982; Panasyuk and Vlasova, 1981; Spjeldvik and Fritz, 1978, 1981, 1983; Sheldon and Hamilton, 1993; Chen et al., 1994, 1996a,b; Selesnick and Mewaldt, 1996; Spjeldvik, 1996b; Pugacheva et al., 1996; and others). For radiation belt protons, there exist empirical models compiled by NASA as well as a survey of proton pitch angle anisotropies with local time, energy and $L$-shell (e.g., Garcia and Spjeldvik, 1985). But for radiation belt ions heavier than hydrogen, detailed data to carry out a local time variation assessment have until now not been available. Using data from the well-instrumented POLAR spacecraft in the NASA/ISTP program, we here study details of the $\mathrm{MeV}$ helium ion anisotropies and azimuthal asymmetries in the radiation belt region.

In an earlier conference paper we have reported on azimuthal helium ion asymmetries in the radiation belts (Spjeldvik et al., 1998), showing shifts in the radial location of the helium ion flux maxima and observed variations in helium ion flux intensities with magnetic local time. In this work we investigate the character of the observed angular anisotropies of hundreds of $\mathrm{keV}$ and several $\mathrm{MeV}$ helium ions. The CAMMICE/HIT instrument does not significantly distinguish between ${ }^{3} \mathrm{He}$ and ${ }^{4} \mathrm{He}$, so our results pertain to the overall helium ion population.

\section{Helium ion observations}

The POLAR spacecraft was launched on February 24, 1997 , and it achieved a near polar orbit with an initial inclination $\sim 86^{\circ}$ to the equator, an initial perigee of $\sim 1.8$ Earth radii over the Earth's south pole, an initial apogee of $\sim 9$ Earth radii over the north pole, and a spacecraft spin time scale of six seconds. Among the extensive instrumentation on the POLAR spacecraft is the CAMMICE instrument package which contains the heavy ion telescope (HIT) consisting of a stack of solid state detectors and electronic discriminators. HIT is mounted perpendicular to the spacecraft spin axis where it operates with an angular and temporal resolution of 16 sector samples per spacecraft spin period. The spacecraft spin axis orientation is nominally perpendicular to the orbital plane such that instruments with pointing direction perpendicular to the spin axis sample virtually all directions angular to the geomagnetic field in each spin as POLAR crosses the geomagnetic equatorial plane. We here report observations of geomagnetically trapped helium ions in four energy ranges (data channels HID5-8) from 520 to $8200 \mathrm{keV}$ ion kinetic energy. These are specified in Table 1. The geometric factor of the CAMMICE/HIT instrument is $\mathrm{g}=9.13 \times 10^{-3} \mathrm{~cm}^{2} \mathrm{sr}$, and the HIT aperture opening angle is $\pm 8^{\circ}$. Except for the highest energy channel which extends up to $8200 \mathrm{keV}$, these POLAR helium ion channels are similar in energy coverage and functionality to the helium ion detector channels, $\Delta \mathrm{a} 1, \Delta \mathrm{a} 2, \Delta \mathrm{a} 3$ and $\Delta \mathrm{a} 4$ utilized on the previous Explorer-45 spacecraft in magnetospheric near-equatorial orbit (e.g. Fritz and Spjeldvik, 1978, 1982).

The POLAR spacecraft made particle and field observations during the low solar activity in the spring, summer and autumn of 1996, and POLAR continues to be operational as of this writing. In the interior of the radiation belts, this time interval constitutes a rather long period of geomagnetic quiescence with the $D_{s t}$ ring current activity index rarely depressed by more than a few tens of nanoTesla between a minor magnetic "storm" on January 13 (with provisional minimum $\left.D_{s t}=-88 \mathrm{nT}\right)$ and another "storm" on October 23, 1996 (with provisional minimum $D s t=-110 \mathrm{nT}$ ). Thus the period from the launch of POLAR to the October 1996 storm represents an opportunity to study the 
Table 1. CAMMICE/HIT

helium ion channels

\begin{tabular}{lllll}
\hline $\begin{array}{l}\text { HIT data } \\
\text { channel } \\
\text { ID }\end{array}$ & $\begin{array}{l}\text { HIT energy } \\
\text { response range } \\
(\mathrm{MeV})\end{array}$ & $\begin{array}{l}\text { Detector half } \\
\text { opening angle } \\
\text { (degrees) }\end{array}$ & $\begin{array}{l}\text { Azimuthal } \\
\text { sweep angle } \\
\text { (degrees) }\end{array}$ & $\begin{array}{l}\text { Geometric } \\
\text { factor: G } \\
\text { (cm }\end{array}$
\end{tabular}

Note. Channel HID5 is also marginally sensitive to protons (from dual proton pile-up), and is not to be used to establish helium ion spectral shapes when such pile-up is present detailed structure of the relatively undisturbed ion radiation belts during sunspot cycle minimum conditions.

\section{Angular and orbital data sampling}

The POLAR CAMMICE/HIT instrument accumulated counts in the solid state detector channels with a resolution of 16 accumulation samples per spacecraft spin. Directionally, this means that each count sample sweeps out $22.5^{\circ}$ of the total spin angular sweep, in addition to the instrument aperture angle of about $\pm 8^{\circ}$. For near-simultaneous on- and off-equatorial observations on essentially the same geomagnetic flux tube, it is possible to gain a complementary view of the local pitch angle distribution that corresponds to an expanded resolution of the distribution at low equatorial pitch angles. The schematic illustration in Fig. 1 shows the parametrized trajectory of the POLAR spacecraft on July 28, 1996 in terms of the ephemeris $L$-parameter, the geomagnetic latitude (MLAT), and the geomagnetic local time (MLT). It can be seen that on its inbound leg

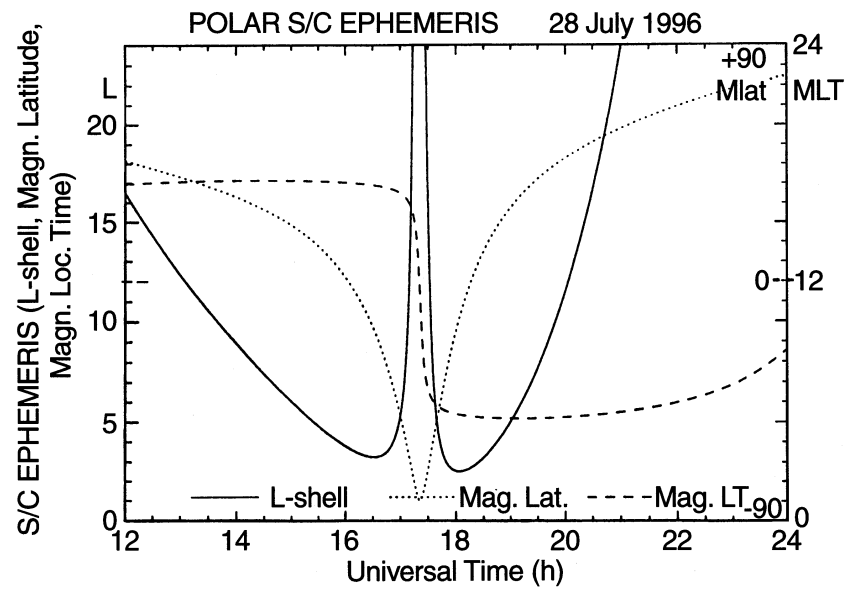

Fig. 1. ISTP/GGS POLAR spacecraft ephemeris parameters for the period UT $=12$ to $24 \mathrm{~h}$ on July 28, 1996. The solid curve depicts the nominal ephemeris $L$-shell, the dotted curve the geomagnetic latitude, MLAT, and the dashed curve the geomagnetic local time, MLT, of the instantaneous spacecraft position. During this period, the POLAR spacecraft passed through the geomagnetic equatorial plane twice, first just after UT $=16: 00 \mathrm{~h}$ near $L=3.2$, and then again after $\mathrm{UT}=18: 00 \mathrm{~h}$ near $L=2.4$. Notice that "minimum in $L$-shell" is not the same at orbital perigee
POLAR first crosses the geomagnetic equator $($ MLAT $\sim 0)$ at about UT $=16: 00 \mathrm{~h}$ just below $L=4$, and since it orbits essentially in the plane of the local geomagnetic field lines, POLAR then exits from the trapping region a few tens of minutes later by traversing virtually the same flux tubes at off-equatorial latitudes at about UT $=16: 40$. The subsequent path of POLAR (during UT $=17: 40$ to 18:30) cuts through the radiation belts again on the other side of the Earth, and this traversal occurs in reverse order at approximately conjugate geomagnetic local times after POLAR having swung over the south pole of the Earth. There are two " $L$-shell minima" (but not orbital perigees) on either side of the Earth (first at MLT $17 \mathrm{~h}$ and then at MLT $\sim 05 \mathrm{~h}$ ).

The $L$-shell parameter used here is the spacecraft ephemeris $L$-value computed from the IGRF 95 model geomagnetic field. A more physical $L$-parameter for specific epochs, varying geophysical current systems and event situations is being developed elsewhere (Richard Selesnick, personal communication, 1997), and that improved $L$-parameter may conceivably organize spacecraft data better, particularly at high $L$-shells in the auroral zone and beyond. However, within the quiet time trapping region there appears to be little difference between the nominal and the specific epoch $L$-parameters as calculated by R. Selesnick. Consequently, this work uses the nominal $L$-parameter for data organization deep within the radiation belts.

To establish the helium ion pitch angle distributions, the available magnetic field direction information (magnetic field elevation angle and azimuth angle in despun spacecraft coordinates) provided in the routine spacecraft ephemeris once per spin period was used. For quiet time interior radiation belt observations, at $L=2.5$ to 7 , this routine six second resolution magnetic field direction information is deemed adequate since temporal variations in the magnetic field on time scales of the spacecraft spin period were either virtually absent or insignificant during this geomagnetically very calm period.

A set of examples of the directly observable angular distributions from the July 28, 1998 passage of POLAR through the radiation belt region at UT $=18: 35$ close to the geomagnetic equator at $L \sim 3$ is presented in Fig. 2. These distributions are single-spin "snapshots" of the observed CAMMICE/HIT dual half-spin counts per spin sector in data channels HID5-8. With orbital plane nominally parallel to the plane of ideal dipole 

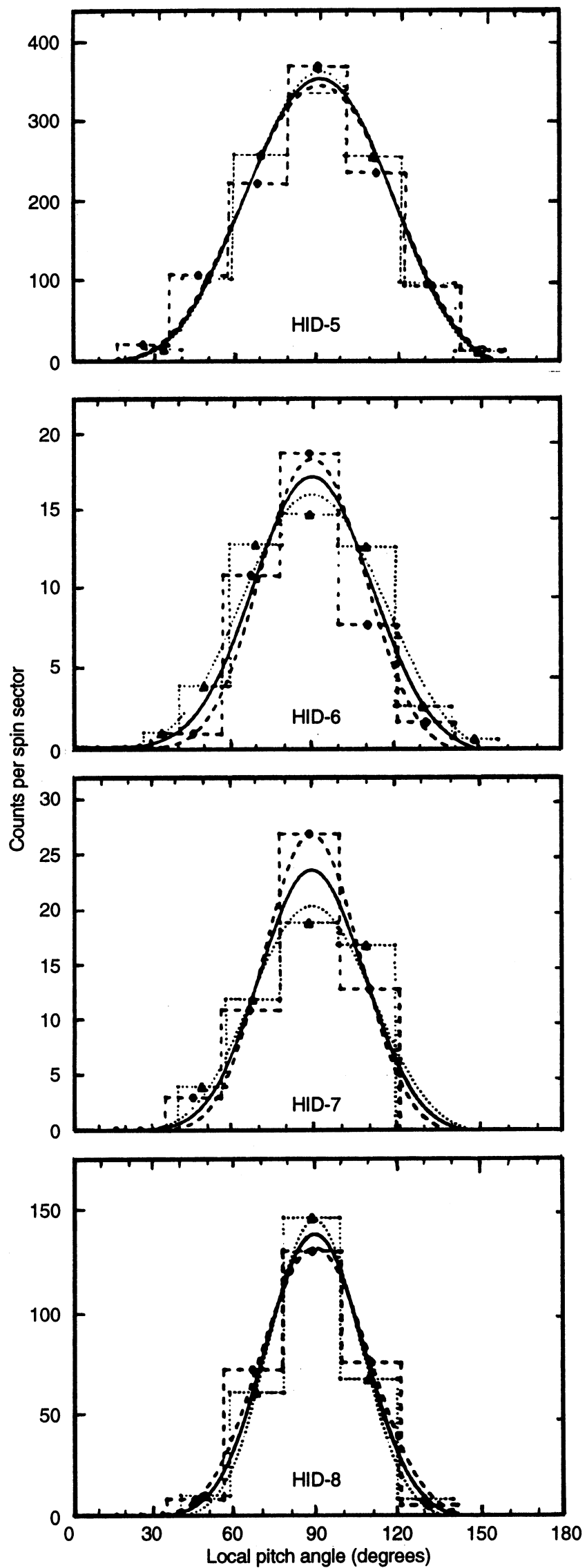

Fig. 2. Observed ISTP/GGS POLAR observations of the helium ion pitch angle distributions in helium ion data channels HID5-8 obtained at $L \sim 3$ and at UT $=18: 25 \mathrm{~h}$ on July 28, 1996 for $B / B_{0}$ $\sim 1.1$. The dashed line histograms are for the magnetospheric radially outward looking half-spin and the dotted histograms are for the inward looking half-spin. The corresponding smooth curves are local $\sin ^{N}(\alpha)$ fits to these angular ion distributions, and the solid curve is the mean of the two

geomagnetic field lines and the POLAR spin axis nominally perpendicular to the orbital plane, it is possible to sample ion distribution gradients in $L$-shell. Due to the large gyro radii of energetic heavy ions, it is possible that radial gradients present in the spatial ion distributions can give rise to different count rates when the instrument detects ions that come from the upgradient direction compared to those that come from the down-gradient direction (inward and outward looking detector aperture), and this effect will be superimposed on the normal statistical fluctuations in the helium ion count sampling. The dashed pitch angle histograms show observed counts for magnetospheric radial outward pointing of the HIT-instrument and dotted histograms are for magnetospheric radially inward recordings (considering the locations of the ion gyro centers), and the average of these count rates would approximate the helium ion flux intensity at the spacecraft location.

Also shown in Fig. 2, the corresponding smooth curve fits to the data versus local pitch angle, $\alpha$, represent least mean-square best fits $\sin ^{N}(\alpha)$ distributions for each half-spin, and the smooth solid line fit is the average of these two fits. The empirically determined exponent, $N$, represents an overall measure of the pitch angle anisotropy, commonly known as the pitch angle anisotropy index, although as we shall see, this single parameter is an incomplete descriptor for the entire equatorial pitch angle distribution. As one would reasonably expect in the heart of the radiation belt confinement region, these are indeed geomagnetically stably trapped distributions exhibiting angular maxima for locally mirroring ions. Since the POLAR spacecraft crossed the geomagnetic equator near the $L$-shell of these data samples, these local helium ion pitch angles, $\alpha$, are also essentially the equatorial pitch angles, $\alpha_{0}$. Thus, the observed peaks in the count rates are not local peaks off the geomagnetic equator, but in fact the actual peaks of the entire equatorial helium ion pitch angle distribution.

The observed anisotropy index $N$-values that one may extract from the fits in this figure and in similar plots are generally found to vary with instrumental energy pass band from about $N \sim 4$ at $\mathrm{E}=0.52$ $1.15 \mathrm{MeV}$ to $\mathrm{N} \sim 9$ at $\mathrm{E}=2.4-8.2 \mathrm{MeV}$. The exemplified trend of finding higher pitch angle anisotropies with higher helium ion energies is qualitatively similar to the observed statistical 1972 helium ion data result from Explorer-45 (Spjeldvik and Fritz, 1978). But quantitatively, the deduced helium ion $N$-values in this 1996 data sample are smaller than the corresponding 1972 result from two solar cycles earlier when the empirical relation: 
$N=7+9.1 \ln \left(\mathrm{E} / \mathrm{E}_{0}\right)$ with $\mathrm{E}_{0}=1 \mathrm{MeV}$ was found (i.e., Fritz and Spjeldvik, 1981). The reason for the difference in these results may be related to the different observational epoch and possibly also to the different magnetic local times of the sampling (the Explorer-45 results were predominantly from the noon-to-early-dusk MLT sector, while the POLAR results on July 28, 1996 are from the dawn-dusk plane).

\section{Mapping to the geomagnetic equator}

From a spacecraft orbit point of view, near-equatorial observations are only possible over a limited $L$-shell range around the POLAR crossing of the geomagnetic equator. Yet it is desirable to obtain reasonably reliable estimates of the equatorial distributions and flux magnitudes over all $L$-shells for which sufficient helium ion count statistics are available to do so. Any energetic particle distribution function, $f$, can be expanded in a series of base functions. From some theoretical considerations, power series, Bessel functions, trigonometric functions, Gaussian distributions, or other special functions may often seem desirable to describe the angular dependence. It is also possible to expand the pitch angle distribution in a power series in sine and cosine functions of the local particle pitch angle, $\alpha$ : $f(\alpha)=\Sigma_{i=0}^{\infty}\left[g_{i} \sin ^{i}(\alpha)+h_{i} \cos ^{i}(\alpha)\right]$ where $g_{i}$ and $h_{i}$ are constants to be empirically determined.

In a trapped energetic ion distribution exhibiting symmetry around a dominant peak at $\alpha=90^{\circ}$, one might expect the terms proportional to $\cos ^{i}(\alpha)$ to be relatively much smaller than the sinusoidal terms. For mathematical simplicity, one might set $h_{i}=0$ for all $i$ index values, and the remainder of the sum is most often approximated by a single dominant term with $i=N$ so that one assumes $\left|g_{i}\right| \ll\left|g_{N}\right|$ for $i$-index values other than $i=N$. In earlier literature, this use of a single term had either not been reasoned at all or was sometimes posteriorily "justified" by goodness of the empirical fits, especially when detailed information to conclude otherwise was not available. From Liouville's theorem it follows that if the equatorial pitch angle distribution truly follows a $\sin ^{N}\left(\alpha_{0}\right)$ form, then the local pitch angle distribution likewise follows a $\sin ^{N}(\alpha)$ form. Of course, there is no compelling reason why a single term sinusoidal power relation should be a complete anisotropy descriptor, and the one-term trigonometric representation is a practical idealization that is at best an approximation suggested empirically by the data. Nevertheless, it is one representation that is most frequently used to describe geomagnetically trapped energetic ion distributions in terms of a single anisotropy index.

When this functional form is assumed a priori, then the relation between local and equatorial quantities appears particularly simple: $f_{0}\left(\alpha_{0}\right)=f_{0}\left(\alpha_{0}=\pi / 2\right)$ $\sin ^{N}\left(\alpha_{0}\right)$ and $f(\alpha)=f(\alpha=\pi / 2) \sin ^{N}(\alpha)$ (e.g., Roederer, 1970). Here $f_{0}\left(\alpha_{0}\right)$ and $f(\alpha)$ are the equatorial and the local pitch angle distribution functions on the same geomagnetic flux tube. By Liouville's theorem, $f_{0}\left(\alpha_{0}\right)=$ $f(\alpha)$, and the local and equatorial pitch angles are connected by the geomagnetic mirror equation. Based on this, the mapping from locally observed distributions to equatorial distributions in a dipolar magnetic field becomes a simple approximation for the distribution function: $f_{0}\left(\alpha_{0}=\pi / 2\right)=f(\alpha=\pi / 2)\left[B / B_{0}\right]^{N / 2}$, where $B$ and $B_{0}$ are the local and equatorial magnetic field induction values on the same geomagnetic flux tube. The mapping is similar for particle count accumulations, count rates (CPS) and particle fluxes (J).

Since we cannot, with a single spacecraft, simultaneously measure both $B$ and $B_{0}$, it is necessary to take the $B / B_{0}$-ratio from a magnetic field model. Well within the interior of the Earth's radiation belts one may use the IGRF 95 model, although not too much inaccuracy in the ratio-values is suffered by using the simple dipole ratio: $B / B_{0}=\left(4-3 \cos ^{2} \lambda\right)^{1 / 2} / \cos ^{6} \lambda$ where $\lambda$ is the instantaneous geomagnetic latitude of the spacecraft. But the simplicity conveyed here is quite alluring since it assumes and depends on the specific functional form of the pitch angle distribution. In fact, mapping to the geomagnetic equator from high $B / B_{0}$-values can go astray if the actual angular distributions deviate significantly from the simple one-term trigonometric representation form. In the following exposition we explore these aspects from an empirical point of view.

As noted, the characteristics of the POLAR orbital trajectory can be used to examine the helium ion pitch angle distribution from two different observational points of view. The top panel of Fig. 3 exemplifies the pitch angle distribution results in one of the CAMMICE/HIT channels, helium channel HID5 ( $\mathrm{E}=0.52$ $1.15 \mathrm{MeV}$ ) at $L=4-4.25$ for the near-equatorial parts of the spacecraft trajectory at UT $=15: 50-15: 55$ on July 28,1996 where $B / B_{0} \sim 1.06$. Fitting a sinusoidalpower function to the distribution yields $N \sim 3.8$, and at first glance the functional fit based on least mean squares deviation from the data appears reasonably good (solid curve is an unweighted fit and the dotted curve is a fit weighted by a factor proportional to the data values themselves). But the apparent good fit can be deceptive, however, for it is in both fitting procedures dominated by the near-equatorial count accumulations. Indeed, if one looks more closely, it becomes apparent that the fits are not quite as good for low equatorial pitch angles where essentially all count accumulations within the indicated pitch angle selection bins systematically fall above the sinusoidal fit-curve. This is a clear indication that the simple one-term functional form sinusoidal distribution does not fully describe the helium ion pitch angle distribution down to low equatorial pitch angles.

As the POLAR spacecraft proceeded to travel on a more off-equatorial trajectory (at UT $=16: 30$ to 18:00), it encountered virtually the same geomagnetic flux tubes and was afforded off-equatorial views of the same flux tube helium ion distributions at $L=4-4.25$ with a much higher $B / B_{0}$-value of $\sim 8$. The corresponding local helium ion angular distribution is depicted in the lower panel of Fig. 3, and it shows a much lower effective angular anisotropy where the local single-term sinusoidal functional fit to the observed pitch angle distribution 


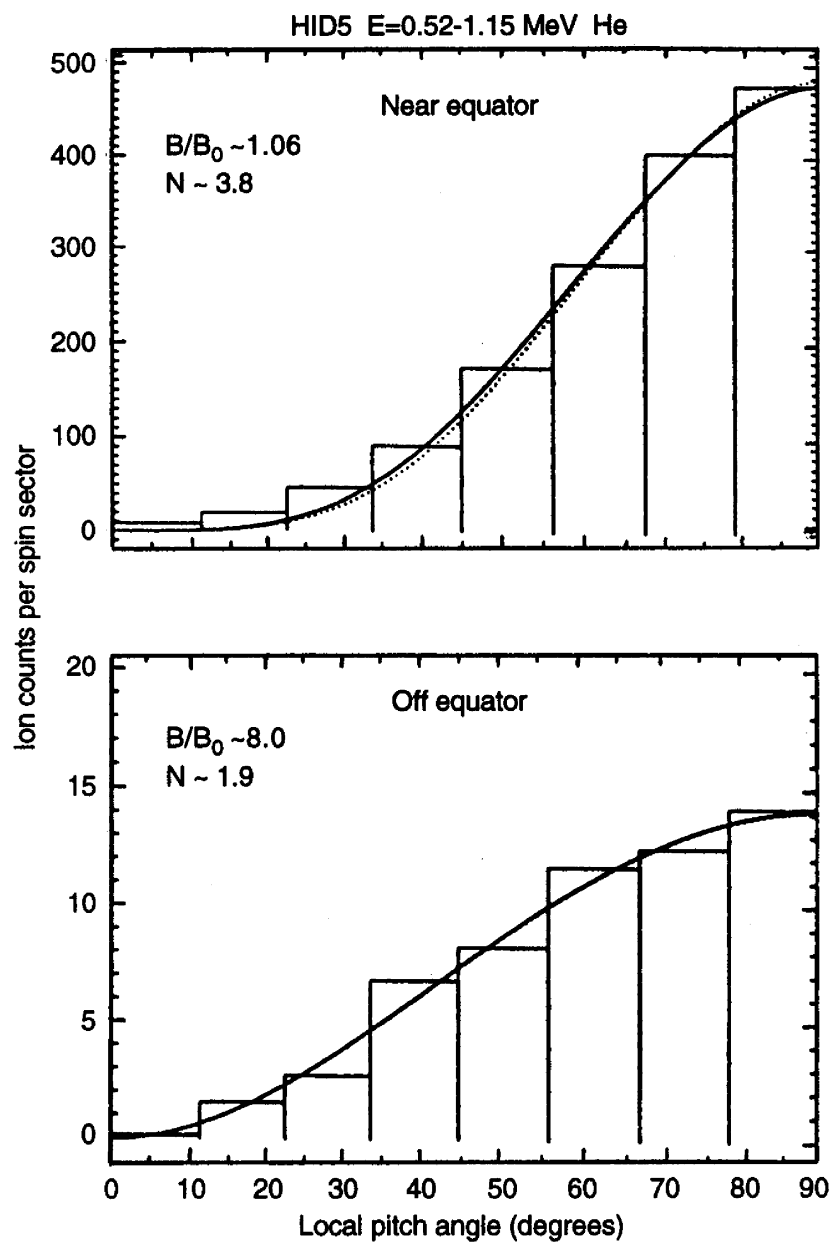

Fig. 3. Helium ion pitch angle distributions observed in the CAMMICE/HIT HID5 data channel at $L=4-4.25$ on July 28, 1996. The data for the UT $=15: 38$ to $15: 43$ when the spacecraft ephemeris indicated $B / B_{0}$-values around 1.06 are depicted in the upper panel yielding a near-equator anisotropy $N$-value of 3.9, and the data for UT $=15: 50$ to $15: 55$ correspond to off-equator $B / B_{0}$-values of about 8 , and yields an off-equatorial $N$-value of 1.9. Similar anisotropy index differences observed on essentially the same flux tubes are also found in the other POLAR HID helium ion data channels as well

yields $N=1.9$, about half of the anisotropy index-value exhibited by that flux tube at the geomagnetic equator.

From the specific quantitative results exemplified already, the entire equatorial helium ion pitch angle distribution in the interior of the radiation belts clearly cannot be described by a single anisotropy index, $N$. This situation is repeatedly encountered in the four energy channels our data set, and it brings about a sobering cautionary view regarding the use on any single "anisotropy index" to fully describe radiation belt ion distributions. The distributions are more complicated than the simplest one-term "theoretical" description would suggest. Indeed, we find this result in all of the POLAR CAMMICE/HIT helium ion data channels and over a wide range of $L$-shells. Consequently, this observed flattening of the angular distribution below an equatorial pitch angle of about $\alpha_{0} \sim 45^{\circ}$ must be a characteristic feature of the helium ion pitch angle anisotropy at the lower equatorial pitch angles for radiation belt helium ions, at least within the upper $\mathrm{keV}$ and lower $\mathrm{MeV}$ energy range.

Another way to extract ion pitch angle distribution information is to convert local pitch angles, $\alpha$, to equatorial pitch angles, $\alpha_{0}=\operatorname{arc} \sin \left[\left(B / B_{0}\right)^{1 / 2} \sin \alpha\right]$ using model $B / B_{0}$-values, and to plot $f_{0}\left(\alpha_{0}\right)=f(\alpha)$ from this. The draw-back of this technique is that the full equatorial pitch angle distribution is not observable from off-equatorial locations, and one is often left with an incomplete distribution exhibiting a gap around $\alpha_{0}=90^{\circ}$. Figure 4 shows an example of the results from this technique. The upper panel in this figure depicts the angular distribution in the HID5 channel $(\mathrm{E}=0.52$ $1.15 \mathrm{MeV}$ ) observed at $L=3.81$ on the same July 28, 1996 POLAR pass with $B / B_{0} \sim 1.004$, the middle panel shows the corresponding result at $L=3.34$ with $B / B_{0}=1.14$, and the lower panel illustrates the situation at $L=3.27$ with $B / B_{0}=1.76$. Inward looking (reconed by gyro center location) half-spins correspond to dashed curves and data values with up-ticks, and outward looking half-spins are shown as dotted curves and data values with down-ticks. The solid smooth curve is the mean of the two half-spin fits to the distributions, and would be representative of the distribution at the spacecraft location.

As one can glean from Fig. 4, the range of unobservable equatorial pitch angles, $\alpha_{0}$, widens considerably with increasing $B / B_{0}$-values so that a "fill-in-fit" of a functional form $\sin ^{N}\left(\alpha_{0}\right)$ becomes impractical and quite unreliable when $B / B_{0}>2$. On the other hand, $B$ field model-based conversion to equatorial pitch angles for the use of equatorial pitch angles for binning pitch angle distribution data in energy, $L$-shell and magnetic local time is, of course, valuable in an effort to produce statistical average distributions based on long-term sampling of steady state distributions.

\section{Observations of anisotropy variations with location}

Even though the "anisotropy $N$-index" has been found to be at best an incomplete measure for the entire helium ion pitch angle distribution, it may still be utilized as a coarse overall descriptor of approximate validity for high equatorial pitch angles, at roughly $\alpha_{0} \sim 90^{\circ} \pm 45^{\circ}$, and to approximately map the near-equatorial fluxes to the geomagnetic equator when $B / B_{0}<2$. To obtain an overview of the variation of the helium ion pitch angle distribution with $L$-shell and to explore any dawn-dusk differences, we studied the pitch angle anisotropy variation over the central section of the radiation belts where energetic helium ions are sufficiently plentiful to allow meaningful angular sampling and for those orbital segments of the POLAR spacecraft where $B / B_{0}<2$.

A juxtaposition of pitch angle anisotropy variation with the $L$-parameter is presented in Fig. 5. This figure depicts the dusk (MLT $\sim 17 \mathrm{~h}$; UT $=14: 45-16: 30)$ and dawn (MLT $\sim 05 \mathrm{~h}$; UT $=18: 00$ to $19: 45$ ) sector radial profiles of the $N$-index deduced (with the restrictions noted above) for the four energy pass bands of the 

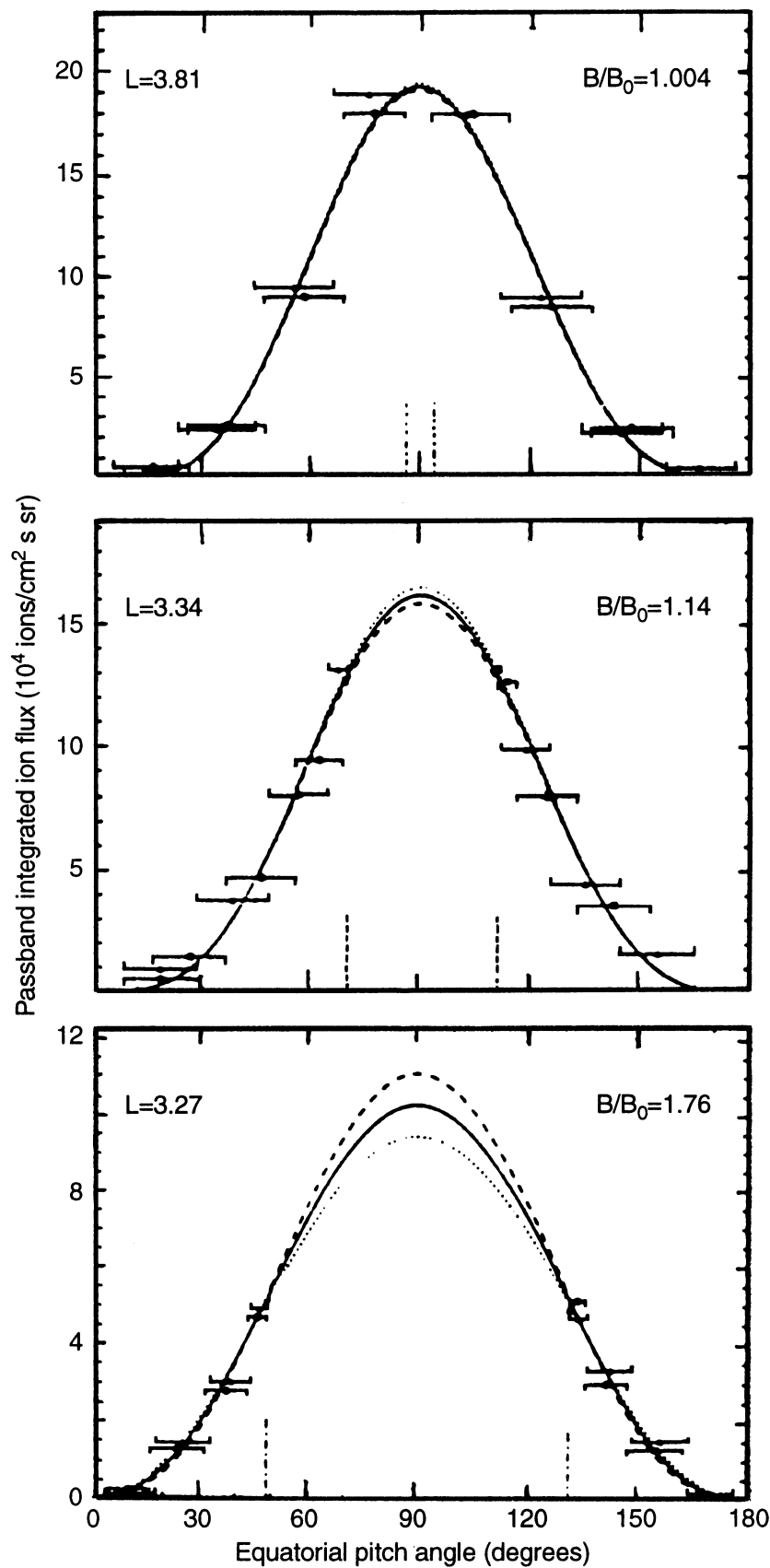

Fig. 4. POLAR observations of helium ion fluxes in the HID5 channel plotted versus equatorial pitch angle, $\alpha_{0}$. The three panels depict distributions over a complete spacecraft spin at $L=3.81,3.34$ and 3.27 with $B / B_{0}=1.004,1.14$ and 1.76 respectively. There is an increasing range of unobservable $\alpha_{0}$-values with increasing $B / B_{0}$. The fitted curves assume a $\sin ^{N} \alpha_{0}$ dependence in each spacecraft half-spin, and the solid smooth curve is the mean fit of these two fits

CAMMICE/HIT instrument on July 28, 1996. The bottom panels also indicate the $B / B_{0}$-value of the observation at the given $L$-shell and MLT. It can be seen that the radial profiles of the $N$-values depict somewhat different features in these different local time sectors.

In the dusk sector and at the lowest energies we calculate pitch angle anisotropy $N$-values (by least mean squares deviation fits) between 3 and 6 when the spacecraft is fairly near the equator. There is a slightly higher pitch angle anisotropy around $L=5$. A similar situation is also seen in panels $2-4$ for data channels HID6-8 (with energies in the range 1.15-8.2 MeV) where a small local anisotropy minimum is seen to occur just beyond $L=4$. However, the radial profiles of $N$ values are different in the dawn sector. Here the orbit of the POLAR spacecraft allows sampling down to about $L=2.5$, and these panels show that the helium ion pitch angle anisotropy increases fairly systematically with lower L-shells in all of our four data channels. The HID5-8 channels show lower pitch angle anisotropy $(N \sim 1$ to 2 at $L \sim 5)$ at dawn than at dusk (where $N \sim 4$ to 5 at $L \sim 5$ ), and all the helium ion channels suggest a brief "plateau" around $L \sim 3.5$ in the otherwise negative $N$-versus- $L$ gradient.

In the earlier data report (Spjeldvik et al., 1998), we computed equatorially mirroring fluxes from the locally mirroring fluxes, and we found that there are systematic geomagnetic local time differences in the flux intensities. Specifically, we tested whether or not there might be any temporal evolutionary trends in the helium ion flux data, in overall fluxes or in the shape of the angular distributions. If present, such variability might tend to obscure a study of anisotropy variations with geomagnetic local time based on data from the slow precession of the POLAR spacecraft over several months. We found essentially no systematic temporal variation of the helium ion fluxes over the study interval.

The dawn-dusk differences found in the POLAR energetic helium ion data, together with the lack of a discernible temporal evolutionary trend in the data set, point to the desirability of carrying out an azimuthal survey covering the anisotropy at all magnetic local times. Since the orbital plane of the POLAR spacecraft precessed in magnetospheric coordinates, the spacecraft progressively sampled all local times over the geomagnetically quiet data period April through October 1996, making a complete survey around the Earth feasible. The azimuthal asymmetry analysis was carried out with 40 spacecraft orbits distributed over this time interval. We determined the helium ion anisotropy $N$-values from least-mean-square fits to the near-equatorial local pitch angle distributions, and for control we also carried out fits to the distributions in local-to-equatorial mapped pitch angle whenever feasible.

The result of carrying out the anisotropy analysis for these POLAR spacecraft orbits in different orbital planes as the spacecraft precessed in magnetic local time sectors are shown in Fig. 6. Here the MLT distribution of the near-equatorial $N$-values obtained at $L=4-4.25$ during April through October 1996 is depicted with long vertical error bars indicating less than perfect sinusoindal-power fits to the local distributions. Count rate limitations also account for a larger spread in the scatter plots of deduced $N$-values for the higher helium ion energies.

Generally we find that rather small $N$-values $(N \sim 2)$ are systematically observed in the early dawn sector and in all the four helium ion data channels (HID5-8). In contrast, significantly higher anisotropy $N$-index values 


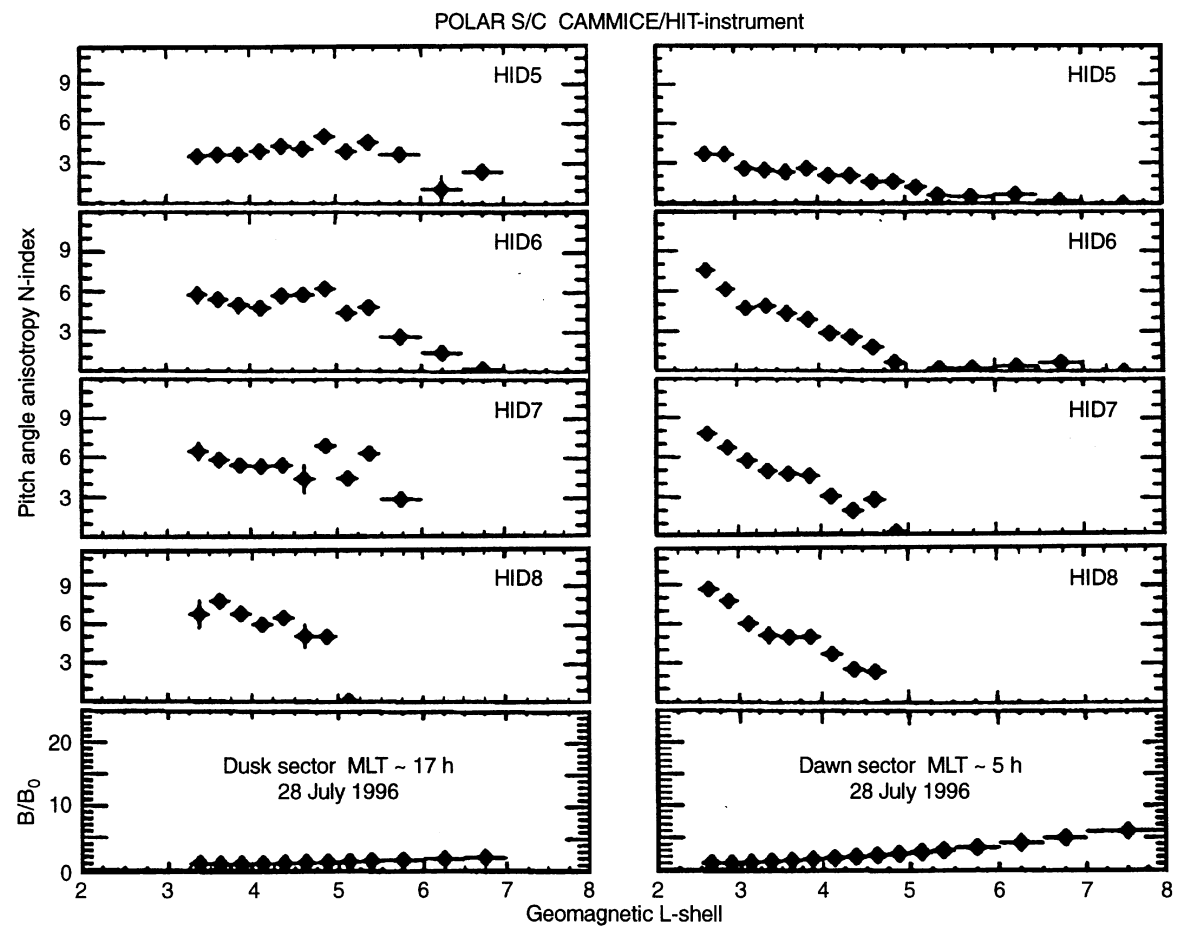

Fig. 5. Left panels: magnetic local time dusk sector variation of the deduced pitch angle $N$-index anisotropy parameter with the $L$-parameter in CAMMICE/HIT data channels HID5-8 together with the representative $B / B_{0}$-values along the POLAR orbit for UT $=14: 00$ to $16: 30$ on July 28 , 1996. Right panels: magnetic local time dawn sector variation of the deduced pitch angle $N$-index anisotropy parameter with $L$-shell and the $B / B_{0}$-values along the POLAR orbit for UT $=18: 00$ to $20: 00$ on that day are observed in the late afternoon sector. These are found to vary significantly with helium ion energy from $N \sim 3.5$ (in channel HID5 at $0.52-1.15 \mathrm{MeV}$ ), $N \sim 4.25$ (in channel HID5 at 1.15-1.8 MeV), $N \sim 5.5$ (in channel HID7 at $1.8-2.4 \mathrm{MeV}$ ), to $N \sim 6$ (in channel HID8 at 2.4-8.2 MeV). Although there is a significant spread in this scatter-plot distribution of helium ion anisotropy $N$ index values, there is a clear systematic variation with magnetic local time that appears unrelated to any temporal evolution of the trapped distribution.

Table 2 gives a more extensive summary of the deduced $N$-values for different energies, $L$-shells and magnetic local times. Beyond the apparent MLT-trends in the data, limitations on count statistics restricts the accuracy of these results when fluxes are low, and this is particularly true in the highest energy data channel which has the lowest count statistics.

\section{Summary and discussion}

Results from the CAMMICE/HIT-detector on the POLAR spacecraft have revealed new detailed information on the angular distributions and magnetic local time structure of radiation belt helium ion anisotropies at energies in the range from 0.52 to $8.2 \mathrm{MeV}$ ion kinetic energy. From these observations we find that:

1. Helium ion fluxes in the upper $\mathrm{keV}$ and lower $\mathrm{MeV}$ kinetic energy range show classic geomagnetic trapping configuration with the highest flux intensities at the geomagnetic equator;

2. A single pitch angle "anisotropy $N$-index" based on an assumed distribution $f_{0}\left(\alpha_{0}\right)=f_{0}\left(\alpha_{0}=p / 2\right) \sin ^{N}\left(\alpha_{0}\right)$ does not describe the entire equatorial pitch angle distribution outside of the atmospheric bounce loss cone, but appears empirically to be at least a fair descriptive measure for equatorial pitch angles, $\alpha_{0} \sim 90^{\circ}$ $\pm 45^{\circ}$. The distributions are found to partially flatten out beyond this angular range;

3. Within the approximate range of descriptive validity during the April through October 1996 epoch, energetic helium ion fluxes are generally anisotropic with simple-measure anisotropy $N$-index values in the range of $N=3$ to 9 , typically increasing with increasing ion energy in the heart of the radiation belt region. The $\mathrm{MeV}$ helium ion anisotropy was systematically found to be higher in the dusk sector than in the dawn sector.

4. Additionally, as earlier reported, although not explicitly exhibited here, equatorial peak helium fluxes are generally found to be located in the vicinity of $L=3$ in this energy range with the higher energies exhibiting radial peaks more Earthward, and the location of the flux peak varied from MLT dawn to dusk sector so that the dawn-side peaks were found at lower $L$-shells than the dusk-side peaks (for details, see Spjeldvik et al., 1998);

Both the geomagnetic and the geoelectric fields are known to cause $L$-shell splitting effects (e.g., Roederer, 1970; Roederer and Schulz, 1971; Stern, 1971; Schulz, 1972; Kivelson and Southwood, 1975a,b), and there will be some effects of these drift shell splitting processes both in the interior of the trapping region and in the outer parts that encounter its boundaries. It is possible that the observed azimuthal asymmetry of $\mathrm{MeV}$ helium ions may be associated with asymmetries in the magnetic field itself and/or with the geoelectric field dawn-dusk structure. Additionally, the Earth has internal magnetic asymmetries that are manifested as higher magnetic multipole contributions with increasing contributions closer to the Earth. But effects of the latter nature 
NASA ISTP/GGS POLAR S/C CAMMICE/HIT-instrument

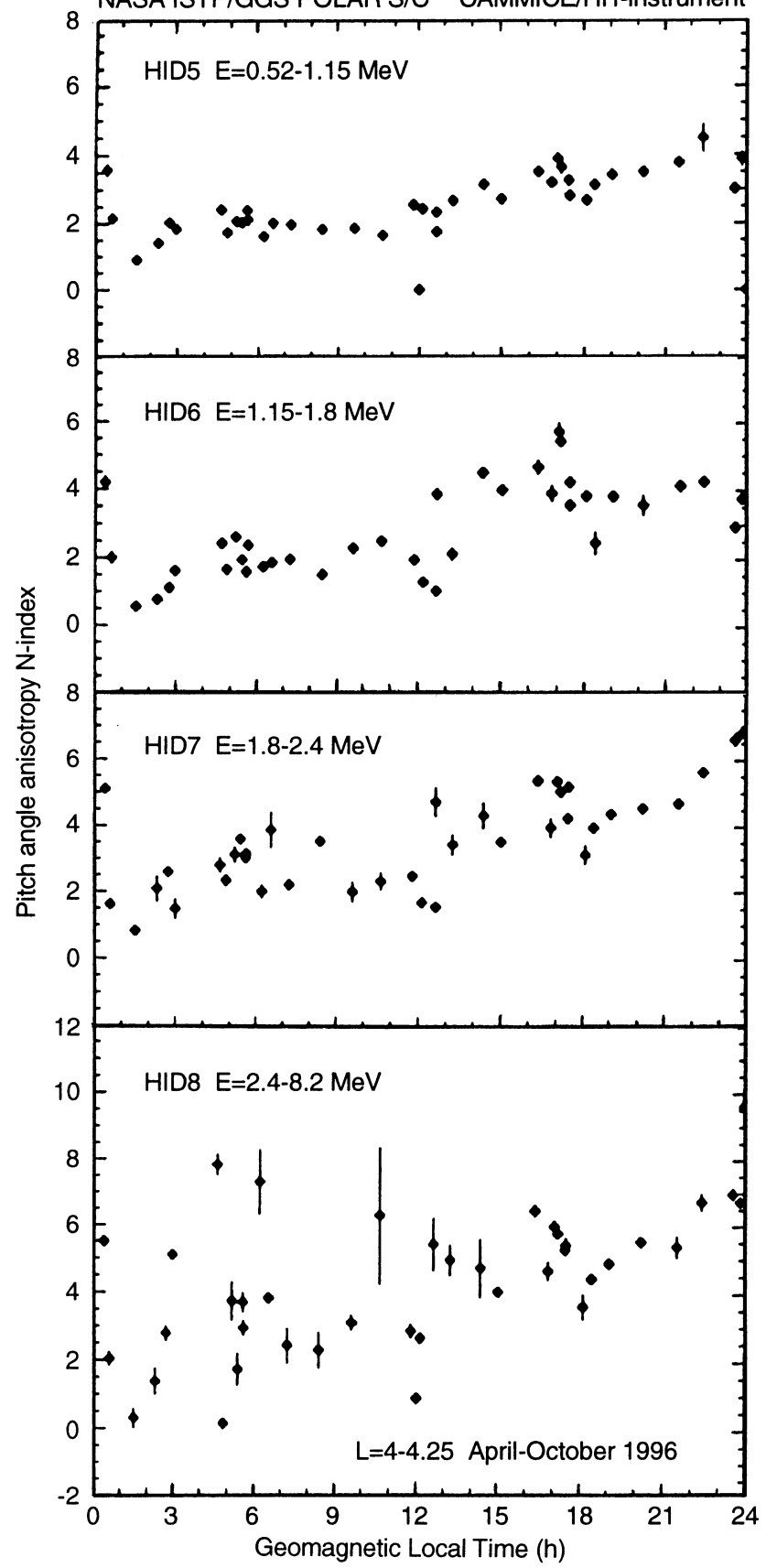

Fig. 6. Distribution in magnetic local time of the deduced nearequatorial helium ion pitch angle anisotropy $N$-index in the four CAMMICE/HIT helium energy channels at 0.52 to $8.2 \mathrm{MeV}$ from data obtained with the POLAR spacecraft during April through October 1996. Notice the larger helium ion pitch angle anisotropies in the MLT dusk sector

should predominantly correlate with the rotation of the planet, not with magnetic local time controlled by the Sun-Earth direction.

It is, in principle, also possible that systematic errors in the magnetic field models (compared with the real field experienced by magnetospheric particles), even during geomagnetically quiet times, could give rise to apparent azimuthal asymmetries in the observed trapped particle distributions. There is at this time no easy way to quantify such a possible error source.

Another possibility is that the observed helium ion azimuthal MLT asymmetry may, in part, be the result of the large spectral and spatial gradients found in the trapped ion fluxes themselves, combined with the rather modest (at best, tens of $\mathrm{kV}$ ) dawn-dusk geoelectric potential field. And if so, then it is even possible that a small shift in the energy spectrum due to this apparently modest effect might appear as a significant flux variation seen with instruments of fixed energy thresholds for ion detection, and so masquerade as anisotropy and peak flux variations. Such an interpretation is mildly supported by the earlier finding that the radial peak fluxes show an azimuthal maximum in the dusk sector for $0.52-1.8 \mathrm{MeV}$ helium, no dusk extremum for 1.8 $2.4 \mathrm{MeV}$ helium, but an azimuthal dusk minimum for 2.4-8.2 MeV helium ions (e.g., Fig. 8 in Spjeldvik et al., 1998).

A more remote possibility for the interior of the radiation belts is that the observed flattening of the energetic helium ion pitch angle distributions for low equatorial pitch angles might possibly be related to the structure of the radially driven source mechanism for these ions. Anderson et al. (1997) have studied nonadiabatic behavior of protons and other ion species in the near-Earth magnetic tail region. It is suggested from this work that the effective source function for protons as well as for helium and other heavy ions would be related to chaotic pitch angle scattering in the neutral sheet as these ions are injected onto geomagnetically stably confined trajectories.

Detailed helium ion combined diffusion-and-drift modeling of these aspects appears desirable to see if a geoelectric field asymmetry effect interpretation for energetic helium ions has any merit in the heart of the Earth's radiation belt region, or if a chaotically driven source process has any significant effect. Conversely, if the observed azimuthal anisotropy variation features should be predominantly the result of errors in the magnetic field model, then the ion observations themselves may be used to probe the magnetic field asymmetry properties in the inner magnetosphere.

Acknowledgements. This work was supported in part by a NASA grant NAG5-2578 to Boston University. The authors are grateful to the entire ISTP/GGS POLAR spacecraft technical management team for data acquisition and routine processing of the telemetry data stream. One of us (WNS) enjoyed the Visiting Scholar guest privileges and hospitality at Boston University during his sabbatical leave from Weber State University. We also want to acknowledge the significant contributions of Bryan Laubscher, Robert Hedges, Rose Vigil, and Gina Lujan on the CAMMICE/HIT sensor system at the Los Alamos National Laboratory; of Rocky Koga, Patricia Lew, Norman Katz, and Bill Crain on the CAMMICE/HIT data processing unit at the Aerospace Corporation; and of the administrative support and interest provided by Donald D. Cobb at the Los Alamos National Laboratory. We also acknowledge valuable Email conversations with Dr. Richard Selesnick at the Aerospace Corporation regarding validity and correction procedures for the $L$-shell coordinate.

Topical Editor K.-H. Glassmeier thanks a referee for his help in evaluating this paper. 
Table 2. Near-equatorial helium ion anisotropy $N$-index

\begin{tabular}{|c|c|c|c|c|c|c|c|}
\hline \multirow{2}{*}{$\begin{array}{l}\text { Channel HID5 } \\
L \text {-shell = } \\
\text { MLT (h) }\end{array}$} & \multicolumn{7}{|c|}{$\mathrm{E}=0.52-1.15 \mathrm{MeV}$} \\
\hline & $2.5-3$ & $3-3.5$ & $3.5-4$ & $4-4.5$ & $4.5-5$ & $5-5.5$ & $5.5-6$ \\
\hline $00-06$ & $3.0 \pm 0.5$ & $2.5 \pm 0.5$ & $2.0 \pm 0.5$ & $2.0 \pm 0.5$ & $1.4 \pm 0.5$ & $1.0 \pm 0.5$ & $0.8 \pm 0.5$ \\
\hline $12-18$ & - & $3.0 \pm 0.5$ & $3.0 \pm 0.6$ & $3.0 \pm 0.7$ & $3.0 \pm 1.0$ & $3.0 \pm 1.2$ & $2.0 \pm 1.0$ \\
\hline $18-24$ & - & - & $3.6 \pm 0.7$ & $3.5 \pm 0.7$ & $2.6 \pm 0.7$ & $2.3 \pm 0.8$ & $1.5 \pm 0.7$ \\
\hline Channel HID6 & \multicolumn{7}{|c|}{$\mathrm{E}=1.15-2.4 \mathrm{MeV}$} \\
\hline $00-06$ & $6.0 \pm 1.5$ & $4.0 \pm 1.2$ & $3.2 \pm 1.2$ & $2.0 \pm 1.0$ & $1.0 \pm 1.0$ & $1.0 \pm 1.2$ & $\sim 0.2$ \\
\hline $06-12$ & $7.0 \pm 2.0$ & $5.0 \pm 2.0$ & $2.8 \pm 1.2$ & $2.0 \pm 0.8$ & $1.5 \pm 1.1$ & $1.0 \pm 0.5$ & $0.7 \pm 0.7$ \\
\hline $12-18$ & - & $5.0 \pm 1.5$ & $4.2 \pm 1.0$ & $4.0 \pm 1.0$ & $4.0 \pm 1.5$ & $2.2 \pm 1.2$ & $1.0 \pm 1.0$ \\
\hline $18-24$ & - & - & $5.0 \pm 0.7$ & $4.4 \pm 1.0$ & $4.0 \pm 0.7$ & $3.5 \pm 1.0$ & $1.5 \pm 1.5$ \\
\hline Channel HID7 & \multicolumn{7}{|c|}{$\mathrm{E}=2.4-4.8 \mathrm{MeV}$} \\
\hline$L$-shell $=$ & $2.5-3$ & $3-3.5$ & $3.5-4$ & $4-4.5$ & $4.5-5$ & $5-5.5$ & $5.5-6$ \\
\hline $18-24$ & - & - & $6.0 \pm 1.0$ & $5.0 \pm 1.5$ & $4.2 \pm 1.2$ & $2.0 \pm 2.0$ & $0.5 \pm 0.5$ \\
\hline Channel HID8 & \multicolumn{7}{|c|}{$\mathrm{E}=4.8-8.2 \mathrm{MeV}$} \\
\hline$L$-shell $=$ & $2.5-3$ & $3-3.5$ & $3.5-4$ & $4-4.5$ & $4.5-5$ & $5-5.5$ & $5.5-6$ \\
\hline $\operatorname{MLT}(\mathrm{h})$ & - & - & - & - & - & - & - \\
\hline $00-06$ & $6.0 \pm 1.0$ & $5.0 \pm 1.0$ & $4.0 \pm 2.0$ & $4.0 \pm 4.0$ & $1.5 \pm 1.5$ & - & - \\
\hline 06-12 & $8.0 \pm 2.0$ & $5.5 \pm 1.5$ & $3.5 \pm 2.5$ & $4.0 \pm 3.0$ & $1.7 \pm 1.5$ & - & - \\
\hline $12-18$ & - & $6.0 \pm 2.0$ & $6.0 \pm 2.0$ & $5.0 \pm 2.5$ & $5.0 \pm 3.5$ & $2.0 \pm 2.0$ & - \\
\hline $18-24$ & - & - & $7.0 \pm 1.5$ & $6.0 \pm 2.0$ & $5.5 \pm 4.0$ & $\sim 1.5$ & - \\
\hline
\end{tabular}

\section{References}

Anderson, B. J., R. B. Decker, and N. P. Paschalidis, Onset of nonadiabatic particle motion in the near-Earth magnetotail, $J$. Geophys. Res., 102, 17 553-17 569, 1997.

Chen. J., T. G. Guzik, Y. Sang, and J. P. Wefel, Energetic helium particles trapped in the magnetosphere, Geophys. Res. Lett., 21, 1583-, 1994.

Chen, J., T. G. Guzik, J. P. Wefel, K. R. Pyle, and J. F. Cooper, Geomagnetically trapped energetic helium nuclei, In: Workshop on the Earth's Trapped Particle Environment, Ed. G. D. Reeves, Am. Ins. Phys. Proc. 383, 161-167, 1996a.

Chen, J., T. G. Guzik, K. R. Pyle, and J. F. Cooper, Energetic helium isotopes trapped in the magnetosphere, J. Geophys. Res., 101, 24 787-, 1996b.

Cornwall, J. M., Radial diffusion of ionized helium and protons: a probe for magnetospheric dynamics, J. Geophys. Res., 77, 1756-, 1972.

Dungey, J. W., Survey of acceleration and diffusion, In: Radiation Trapped in the Earth's Magnetic Field, Ed. B. M. McCormac, D. Reidel, Dordrecht, The Netherlands, 1966.

Fritz, T. A., and W. N. Spjeldvik, Observations of energetic radiation belt helium ions at the geomagnetic equator during quiet conditions, J. Geophys. Res., 83, 2579-2583, 1978.

Fritz, T. A., and W. N. Spjeldvik, Simultaneous quiet time observations of energetic radiation belt protons and helium: the equatorial a/p ratio near $1 \mathrm{MeV}, J$. Geophys. Res., 84, 26082618, 1979.

Fritz, T. A., and W. N. Spjeldvik, Steady state observations of geomagnetically trapped energetic heavy ions and their implications for theory, Planet. Space. Sci., 29, 1169-1193, 1981.

Fritz, T. A., and W. N. Spjeldvik, Pitch angle distributions of geomagnetically trapped $\mathrm{MeV}$ helium ions during quiet times, J. Geophys. Res., 87, 5095-, 1982.

Garcia, H. A., and W. N. Spjeldvik, Anisotropy characteristics of geomagnetically trapped ions, J. Geophys. Res., 90, 347-, 1985.
Gusev, A. A., T. Kohno, W. N. Spjeldvik, I. M. Martin, G. I. Pugacheva and A. Turtelli, Dynamics of the low-altitude energetic proton fluxes beneath the main terrestrial radiation belts, J. Geophys. Res., 101, 19 659-, 1996.

Hamlin, D. A., R. Karplus, R. C. Vik, and K. M. Watson, Mirror and azimuthal drift frequencies for geomagnetically trapped particles, J. Geophys. Res., 66, 1-4, 1961.

Hovestadt, D., B. Hausler, and M. Scholer, Observations of energetic particles at very low altitudes near the geomagnetic equator, Phys. Rev. Lett., 28, 1340-, 1972.

Joselyn, J. A., and L. R. Lyons, Ion cyclotron wave growth calculated from satellite observations of proton ring current decay, J. Geophys. Res., 81, 2275-2283, 1976.

Kivelson, M. G., and D. J. Southwood, Local time variations of particle flux produced by an electrostatic field in the magnetosphere, J. Geophys. Res., 80, 56-65, 1975a.

Kivelson, M. G., and D. J. Southwood, Note on the electric splitting of drift shells, J. Geophys. Res., 80, 3525-3527, 1975 b.

Krimigis, S. M., and J. A. Van Allen, Geomagnetically trapped alpha particles, J. Geophys. Res., 81, 2275-, 1976.

Lyons, L. R., and R. M. Thorne, Equilibrium structure of radiation belt electrons, J. Geophys. Res., 78, 2142-2149, 1973.

Lyons, L. R., and D. S. Evans, The inconsistency between proton charge exchange and the observed ring current decay, $J$. Geophys. Res., 81, 6197-, 1976.

Mogro-Campero, A., Geomagnetically trapped carbon, nitrogen and oxygen nuclei, J. Geophys. Res., 77, 2799-, 1972.

Nakada, N. P., and G. D., Mead, Diffusion of protons in the outer radiation belt, J. Geophys. Res., 70, 4777-, 1965.

Northrop, T. G., and E. Teller, Stability of adiabatic motion of charged particles in the Earth's field, Phys. Rev., 117, 215, 1960.

Panasyuk, M. I., and N. A. Vlasova, Anisotropy of fluxes of protons and alpha particles with energies greater than $4 \mathrm{MeV}$ in the radiation belts, Cosmic Res., 19, 52-, 1981.

Pugacheva, G. I, A. A. Gusev, I. M. Martin, W. N. Spjeldvik, and T. Kohno, The temporal evolution of 3-9 MeV/nucleon He flux 
trapped in the magnetosphere, Geophys. Res. Lett., 23, 2793-, 1996.

Roederer, J. G., Dynamics of geomagnetically trapped radiation, Springer-Verlag, New York Berlin Heidelberg, 1970.

Roederer, J. G., and M. Schulz, Splitting of drift shells by the magnetospheric electric field, J. Geophys. Res., 76, 1055-, 1971.

Schulz, M., Drift shell splitting at arbitrary pitch angle, J. Geophys. Res., 77, 624-, 1972.

Schulz, M., and L. J. Lanzerotti, Particle diffusion in the radiation belts, Springer-Verlag, New York Berlin Heidelberg, 1974.

Selesnick, R. S., and R. A. Mewaldt, Modeling He and H isotopes in the radiation belts, In: Radiation belts: Models and Standards, Geophysical Monograph, 97, 123-128, American Geophysical Union, Washington, D. C. 1996.

Sheldon, R. B., and D. C. Hamilton, Ion transport and loss in the Earth's quiet ring current. 1. Data and standard model, $J$. Geophys. Res., 98, 13491, 1993.

Smith, P. H., and N. K. Bewtra, Charge exchange lifetimes for ring current ions, Space Sci. Rev., 22, 301-318, 1978.

Smith, P. H., N. K. Bewtra, and R. A. Hoffman, Inference of the ring current ion composition by means of charge exchange decay, J. Geophys. Res., 86, 3470-3480, 1981.

Southwood, D. J., and M. G. Kivelson, An approximate analytic description of plasma bulk parameters and pitch angle anisotropy under adiabatic flow in a dipolar magnetospheric field, $J$. Geophys. Res., 80, 2069-2073, 1975.

Spjeldvik, W. N., Expected charge states of energetic ions in the magnetosphere, Space Sci. Rev., 23, 499-, 1979.

Spjeldvik, W. N., Cross field entry of high charge state energetic heavy ions into the Earth's magnetosphere, Geophysical Monograph, 97, American Geophysical Union, Washington, D.C., 97, 63-67, 1996a.

Spjeldvik, W. N., Numerical modeling of stably and transiently confined energetic heavy ion radiation in the Earth's magnetosphere, Radiat. Meas., 26, 309-, 1996b.
Spjeldvik, W. N., and T. A. Fritz, Energetic ionized helium in the quiet time radiation belts: theory and comparison with observation, J. Geophys. Res., 83, 654-, 1978.

Spjeldvik, W. N., and T. A. Fritz, Observations of energetic helium ions in the Earth's radiation belts during a sequence of geomagnetic storms, J. Geophys. Res., 86, 2317-, 1981.

Spjeldvik, W. N., and T. A. Fritz, Experimental determination of geomagnetically trapped heavy ion fluxes, In: Advances in Earth and Planetary Sciences, Ed. R. G. Johnson, 5, 369, 1983.

Spjeldvik, W. N., and P. L. Rothwell, The radiation belts, Chapter 5 In: Handbook of Geophysics and the Space Environment, US Government Printing Office, Washington, D.C., 1985.

Spjeldvik, W. N., G. I. Pugacheva, A. A. Gusev, I. M. Martin, and N. M. Sobolevsky, Sources of inner radiation zone energetic helium ions: Cross-Field transport versus in-situ nuclear reactions, Advances in Space Research, 21, 1675-1678, 1998.

Spjeldvik, W. N., T. A. Fritz, R. B. Sheldon, and J. Chen, Magnetic local time survey of radiation belt helium ions structure conducted with data from the POLAR CAMMICE/HIT instrument, In: Physics and chemistry of the Earth, in press, 1998.

Stern, D. P., Shell splitting due to electric fields, J. Geophys. Res., 76, 7787-, 1971

Taylor, W. W. L., and L. R. Lyons, Simultaneous equatorial observations of 1 - to $30-\mathrm{Hz}$ waves and pitch angle distributions of ring current ions, J. Geophys. Res., 81, 6177-6183, 1976.

Tverskoy, B. A., Dynamics of the Earth's radiation belt region, NASA Technical Translation, TTF-635, Washington, D.C., 1971.

Walt, M., Introduction to geomagnetically trapped radiation, Cambridge University Press, New York, 1994. 